\title{
Edge Diffraction of a Convergent Wave
}

\author{
Alexander C. Livanos and Nicholas George
}

\begin{abstract}
Closed-form solutions have been derived for the diffraction patterns at the focal plane of (1) a convergent wave of unit amplitude illuminating a segment of a circular aperture and (2) a convergent wave of Gaussian amplitude diffracted by an infinite edge. Photographs showing the main features of these edge transform patterns are presented together with computer-generated graphs.
\end{abstract}

\section{Introduction}

The diffraction of an electromagnetic wave by an edge is a basic problem in optics. Prior to Sommerfeld's rigorous solution of this problem for illumination by a plane wave in 1896 , it was customary to consider an infinite edge as a limiting form of the solution for a rectangular aperture illuminated by a plane wave. In this earlier formulation, Kirchhoff's assumption is made as to the aperture distribution; the result is expressed in terms of Fresnel integrals and interpreted physically using Cornu's spiral. ${ }^{1}$ More recently, Keller has treated this problem using his geometrical theory of diffraction, and he compares his result with Sommerfeld's exact solution. ${ }^{2}$ Related boundary-value-problem solutions for perfectly conducting slits are described by Braunbek and Laukien $^{3}$ and Borgnis and Papas. ${ }^{4}$ Edge diffraction of Gaussian laser beams has been analyzed in the Fresnel zone region by Pearson et al. ${ }^{5}$

With the current interest in optical processing and optical transforms, ${ }^{6}$ it is appropriate to analyze the diffraction of a spherically curved wave incident on an edge, i.e., a semiinfinite, opaque screen. We have not found this problem treated in any detail in the literature, although the related problem of the field distribution at the focus of a lens has been analyzed extensively. ${ }^{7-9}$ A qualitative feeling for the difference between these two optical transforms can be obtained by looking at Fig. 1(a), which shows the intensity recorded in the focal plane of a lens illuminated by a uniform plane wave truncated by a circular aperture. Positioning an edge at the lens so as to reduce the opening to a semicircular aperture leads to

The authors are with the California Institute of Technology, Pasadena, California 91109.

Received 29 July 1974. the intensity distribution shown in Fig. 1(b). The introduction of this edge causes an intense spike of energy to appear in the focal plane, at right angles to the edge, which also has symmetry (in intensity) about the origin in the focal plane.

In the following section we obtain approximate solutions for the focal plane diffraction patterns of (1) a spherically convergent, uniform amplitude wave truncated by a segment of a circular aperture and (2) a spherically convergent, Gaussian beam truncated by an offset edge. These results show that the truncation produces a radial spoke of high intensity, symmetrical about the origin. The intensity has an envelope falling off in proportion to the square of the distance from the center of the pattern, i.e., from the focal point. For the circular aperture the pattern oscillates at an angular spacing consistent with the ratio of the wavelength $\lambda$ to the aperture opening. The Gaussian amplitude taper leads to the same inverse-distance-squared dependence of intensity but there is no ringing when the edge is positioned symmetrically in the beam.

\section{Convergent Wave of Uniform Amplitude}

\section{Theory}

Consider an aperture located in the plane at $z=0$ having a transmittance function $T(\xi, \eta)$ given by

$$
T(\xi, \eta)=\operatorname{circ}\left[\left(\xi^{2}+\eta^{2}\right)^{1 / 2} / a\right]\{1 / 2+[\operatorname{sgn}(\xi-d)] / 2\},
$$

where $(\xi, \eta)$ are Cartesian coordinates, $a$ is the radius, and $d$ is the offset (see Fig. 2). The circ and sgn functions are defined by

$$
\operatorname{circ}\left[\left(\xi^{2}+\eta^{2}\right)^{1 / 2} / a\right]\left\{\begin{array}{l}
=1 \text { when }\left(\xi^{2}+\eta^{2}\right) / a^{2} \leq 1, \\
=0 \text { otherwise, }
\end{array}\right.
$$

and

$$
\operatorname{sgn}(\xi-d) \quad\left\{\begin{array}{l}
=1 \text { when } \xi-d>0, \\
=-1 \text { when } \xi-d<0 .
\end{array}\right.
$$




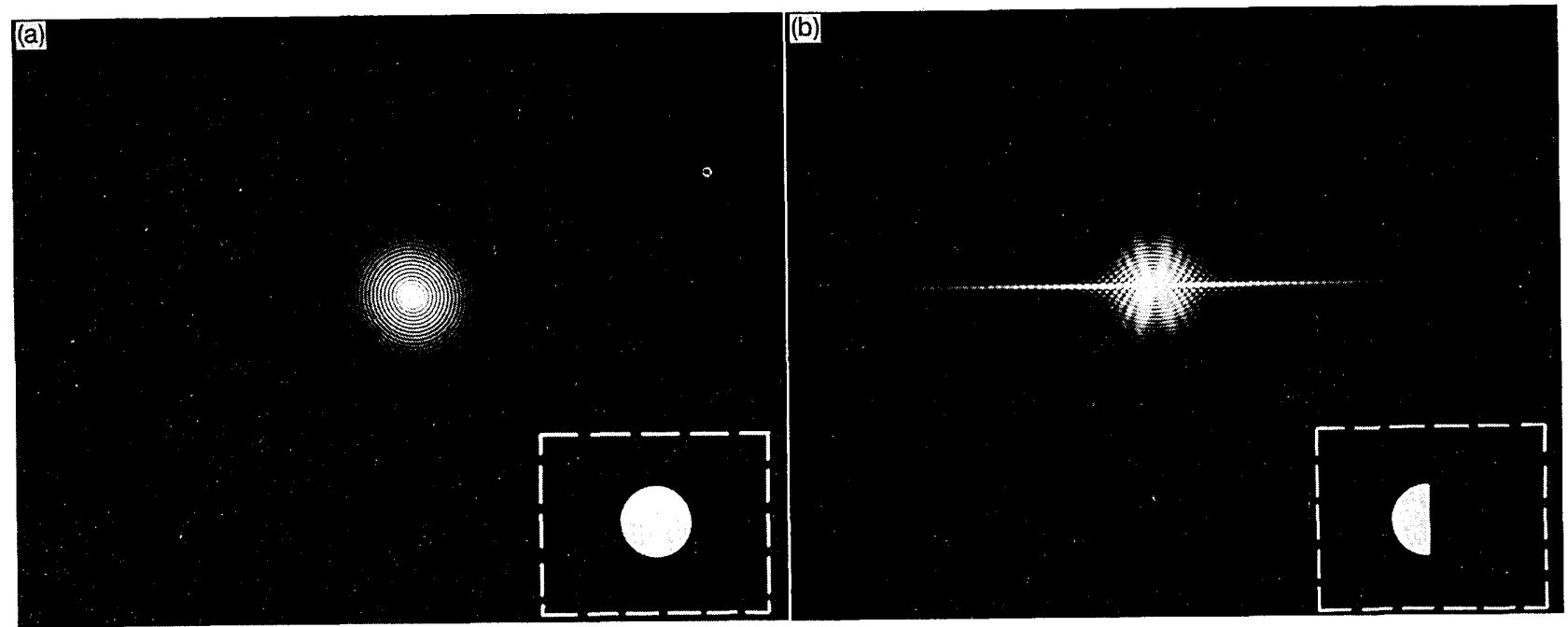

Fig. 1. (a) Radiation pattern at the back focal plane of a lens illuminated by a wave of uniform amplitude and truncated by the circular aperture shown in the inset. (b) Recorded intensity for the same illuminations as for (a) with the addition of an edge at the lens so as to reduce the opening to a semicircular aperture, shown in the inset.

Assume that a monochromatic, plane-polarized spherical wave with radius of convergence $s$ is incident on the aperture. This transverse, scalar component $U(\xi, \eta)$ of the electric field is written in the wellknown paraxial form, suppressing the time dependence and unessential phase terms, as follows:

$$
U(\xi, \eta)=\exp \left[(i \pi / \lambda s)\left(\xi^{2}+\eta^{2}\right)\right] .
$$

The approximate form of Sommerfeld's diffraction theory integral that is appropriate for the calculation of the field $V(x, y, s)$ in the Fresnel-zone region (written for $\exp (i \omega t)$ dependence $)$ is given by

$$
\begin{aligned}
& V(x, y, z=s)=\frac{i \exp [-i 2 \pi s / \lambda]}{\lambda s} \\
& \quad \times \iint_{-\infty}^{\infty} U^{\prime} \exp \left\{-\frac{i \pi}{\lambda s}\left[(\xi-x)^{2}+(\eta-y)^{2}\right]\right\} d \xi d \eta,
\end{aligned}
$$

where Kirchhoff's approximation for the aperture distribution $U^{\prime}$ is to use

$$
U^{\prime}(\xi, \eta)=T(\xi, \eta) U(\xi, \eta) .
$$

Equations (1) and (4) are substituted into Eq. (5) as specified by Eq. (6), giving

$$
\begin{aligned}
V(x, y, z=s)=\{ & i \exp [-i 2 \pi s / \lambda \\
& \left.\left.-i \pi\left(x^{2}+y^{2}\right) /(\lambda s)\right] / \lambda s\right\}\left(I_{1}+I_{2}\right) .
\end{aligned}
$$

The first integral $I_{1}$ is given by

$$
\begin{aligned}
& I_{1}=\frac{1}{2} \iint_{-\infty}^{\infty} \operatorname{circ}\left[\frac{\left(\xi^{2}+\eta^{2}\right)^{1 / 2}}{a}\right] \\
& \times \exp [i 2 \pi(x \xi+y \eta) /(\lambda s)] d \xi d \eta,
\end{aligned}
$$

which is the well-known Airy pattern and is evaluated in Goodman. ${ }^{10}$ The result is

$$
I_{1}=\pi a^{2}\left\{J_{1}\left[\alpha\left(x^{2}+y^{2}\right)^{1 / 2}\right] / \alpha\left(x^{2}+y^{2}\right)^{1 / 2}\right\},
$$

in which $\alpha=2 \pi a /(\lambda s)$ and $J_{1}$ is the order-one Bessel function of the first kind.

The second term of Eq. (7), $I_{2}$, is defined by the following integral:

$$
\begin{aligned}
I_{2}=\frac{1}{2} \iint_{-\infty}^{\infty} \operatorname{circ}\left[\frac{\left(\xi^{2}+\eta^{2}\right)^{1 / 2}}{a}\right] \operatorname{sgn}(\xi-d) \\
\times \exp [i 2 \pi(x \xi+y \eta) /(\lambda s)] d \xi d \eta .
\end{aligned}
$$

It is noted that $I_{2}$ is the Fourier transform of the product of the circ function and an offset sgn function. Physically, this can be interpreted as an open circular aperture with an offset phase mask varying from 0 to $\pi \mathrm{rad}$, stepwise, at $\xi=d$. While using the convolution theorem enables one to express Eq. (10) in terms of a convolution of $J_{1}\left[\alpha\left(x^{2}+y^{2}\right)^{1 / 2}\right] /\left[\alpha\left(x^{2}+\right.\right.$ $\left.\left.y^{2}\right)^{1 / 2}\right]$ with a function proportional to exp $[i 2 \pi x d /$ $(\lambda s)] / x$, we prefer to evaluate this integral in a different manner. First, we integrate with respect to $\eta$. Then we expand the integrands in terms of Bessel functions that can be integrated term by term. The general result thus obtained, Eq. (17), is particularly well suited to numerical calculations.

Integration with respect to $\eta$ gives

$$
\begin{aligned}
I_{2}=\frac{1}{2} \frac{\lambda s}{\pi y} \int_{-a}^{+a} \operatorname{sgn}(\xi-d) \sin & {\left[\frac{2 \pi y}{\lambda s}\left(a^{2}-\xi^{2}\right)^{1 / 2}\right] } \\
& \times \exp [i 2 \pi x \xi /(\lambda s)] d \xi .
\end{aligned}
$$

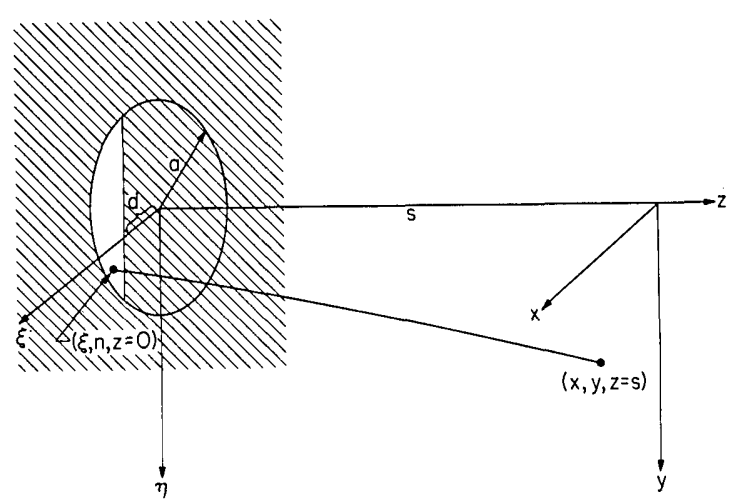

Fig. 2. Coordinate geometry for the aperture, consisting of a segment of a circle, and the back focal plane. 
Substitution of $u=\xi / a$ in Eq. (11) and rearranging terms lead to the following form:

$$
\begin{aligned}
I_{2}=a^{2} \pi \frac{\dot{2}}{\pi \alpha y} & \left\{i \int_{d / a}^{1} \sin \left[\alpha y\left(1-u^{2}\right)^{1 / 2}\right] \sin (\alpha x u) d u\right. \\
& \left.-\int_{0}^{d / a} \sin \left[\alpha y\left(1-u^{2}\right)^{1 / 2}\right] \cos (\alpha x u) d u\right\} .
\end{aligned}
$$

Letting $u=\sin \theta$ in Eq. (12), using the Bessel function identities from Ref. 11, AS9.1.42, 43, 45, and integrating over appropriate limits of $\theta$ yield the following result:

$$
\begin{aligned}
I_{2}=a^{2} \pi & \cdot \frac{2}{\pi \alpha y} \cdot\left\{\sum _ { n = 0 } ^ { \infty } ( - 1 ) ^ { n } J _ { 2 n + 1 } ( \alpha y ) \cdot \left[i \sum_{m=0}^{\infty} J_{2 m+1}(\alpha x) \cdot g_{1}(m, n)\right.\right. \\
& \left.\left.-\sum_{m=0}^{\infty}\left(1-\frac{1}{2} \delta_{0 m}\right)(-1)^{m} J_{2 m}(\alpha x) \cdot g_{2}(m, n)\right]\right\}, \quad \text { (13) }
\end{aligned}
$$

where the Kronecker delta $\delta_{\mathrm{om}}=1$ for $m=0$ and is zero for $m \neq 0$ and $g_{1}, g_{2}$ are given by

$$
\begin{aligned}
g_{1}(m, n)= & \frac{\cos \left[(2 m+2 n+3) \sin ^{-1}(d / a)\right]}{2 m+2 n+3} \\
& \quad+\frac{\cos \left[(2 m+2 n+1) \sin ^{-1}(d / a)\right]}{2 m+2 n+1} \\
+ & \frac{\cos \left[(2 n-2 m+1) \sin ^{-1}(d / a)\right]}{2 n-2 m+1} \\
& +\frac{\cos \left[(2 m-2 n+1) \sin ^{-1}(d / a)\right]}{2 m-2 n+1}, \quad(14) \\
g_{2}(m, n)= & \frac{\sin \left[(2 m+2 n+2) \sin ^{-1}(d / a)\right]}{2 m+2 n+2} \\
& +\frac{\sin \left[(2 m+2 n) \sin ^{-1}(d / a)\right]}{2 m+2 n} \\
+ & \frac{\sin \left[(2 n-2 m+2) \sin ^{-1}(d / a)\right]}{2 n-2 m+2} \\
& \quad \frac{\sin \left[(2 m-2 n) \sin ^{-1}(d / a)\right]}{2 m+2 n} .
\end{aligned}
$$

The general result for the electric field $V$ is found by substitution of Eqs. (9) and (13) into Eq. (7), as follows:

$$
\begin{aligned}
& V(x, y, z=s)=\frac{i}{\lambda s} \exp (-i k s) \exp \left[-i \pi\left(x^{2}+y^{2}\right) /(\lambda s)\right] \pi a^{2} \\
& \times\left(J_{1}\left[\alpha\left(x^{2}+y^{2}\right)^{1 / 2}\right] /\left[\alpha\left(x^{2}+y^{2}\right)^{1 / 2}\right]\right. \\
&+\frac{2}{\pi \alpha y}\left\{\sum _ { n = 0 } ^ { \infty } ( - 1 ) ^ { n } J _ { 2 n + 1 } ( \alpha y ) \left[i \sum_{m=0}^{\infty} J_{2 m+1}(\alpha x) \cdot g_{1}(m, n)\right.\right. \\
&\left.\left.\left.-\sum_{m=0}^{\infty}\left(1-\frac{1}{2} \delta_{0 m}\right)(-1)^{m} J_{2 m}(\alpha x) g_{2}(m, n)\right]\right\}\right),
\end{aligned}
$$

where $k=2 \pi / \lambda$.

A case of particular interest occurs when the edge is symmetrically positioned, i.e., when $d=0$. In this case, Eq. (16) reduces to the following:

$$
\begin{aligned}
V(x, y, z= & s)=\frac{i}{\lambda s} \exp (-i k s) \exp \left[-i \pi\left(x^{2}+y^{2}\right) /(\lambda s)\right] \pi a^{2} \\
& \times\left\{J_{1}\left[\alpha\left(x^{2}+y^{2}\right)^{1 / 2}\right] /\left[\alpha\left(x^{2}+y^{2}\right)^{1 / 2}\right]\right. \\
+ & \left.\frac{2 i}{\pi \alpha y} \sum_{n=0}^{\infty} \sum_{m=0}^{\infty}(-1)^{n} J_{2 n+1}(\alpha y) J_{2 m+1}(\alpha x) g_{0}(m, n)\right\} .
\end{aligned}
$$

Also by Eq. (15), $g_{2}=0$; and by Eq. (14) $g_{1}$ reduces to the form in Eq. (18) that we denote by $g_{0}(m, n)$ :

$$
\begin{aligned}
g_{0}(m, n) & =\{1 /[2(m+n)+3]\}+\{1 /[2(m+n)+1]\} \\
- & \{1 /[2(n-m)+1]\}+\{1 /[2(m-n)+1]\} .
\end{aligned}
$$

The diffraction patterns for this case are discussed in the following section, together with details about the calculations along $y=0$.

\section{Computations and Discussion}

The diffraction pattern for a circular aperture illuminated by a convergent plane wave with uniform amplitude (to within $\pm 4 \%$ ) is shown in Fig. 1(a) and for a semicircular aperture, i.e., $d=0$, in Fig. 1(b).

An interesting method for finding the position of the back focal plane of a lens had been used. The intensity distribution, for unit amplitude and Gaussian waves, near the focal plane of a lens is symmetric about the optical axis. Because of this symmetry the exact determination of the focal plane of the lens is difficult. However, if half the lens is blocked, the intensity patterns are symmetric only at the focal plane, and the above symmetry is very sensitive to translation of the lens along the optical axis.

Numerical evaluation of Eqs. (17) and (18) is shown in Fig. 3 along the major lobe of the radiation pattern. Curve $(A)$ is for the semicircular aperture showing $V V^{*}$ from Eq. (17) normalized by $\left[\pi a^{2} /\right.$ $(\lambda s)]^{2}$ vs $2 \pi a x /(\lambda s)$ with $y=0$. Note that at the origin $I_{1}=0.5$ and $I_{2}=0$; hence the normalized intensity peaks at the value 0.25 .

From Eqs. (7) and (9), we see that for a circular aperture the intensity $V_{0} V_{0} *$ is given by

$V_{0} V_{0}^{*}=\left(\left(\pi a^{2} / \lambda s\right)\left\{J_{1}\left[\alpha\left(x^{2}+y^{2}\right)^{1 / 2}\right] / \alpha\left(x^{2}+y^{2}\right)^{1 / 2}\right\}\right)^{2}$,

where recall, by Eq. (1), this implicitly assumes that the amplitude transmission is only 0.5 across the full aperture. Curve (B) shows $V_{0} V_{0} *$ from Eq. (19) normalized by $\left[\pi a^{2} /(\lambda s)\right]^{2}$ vs the same normalized $x$ abscissa and with $y=0$.

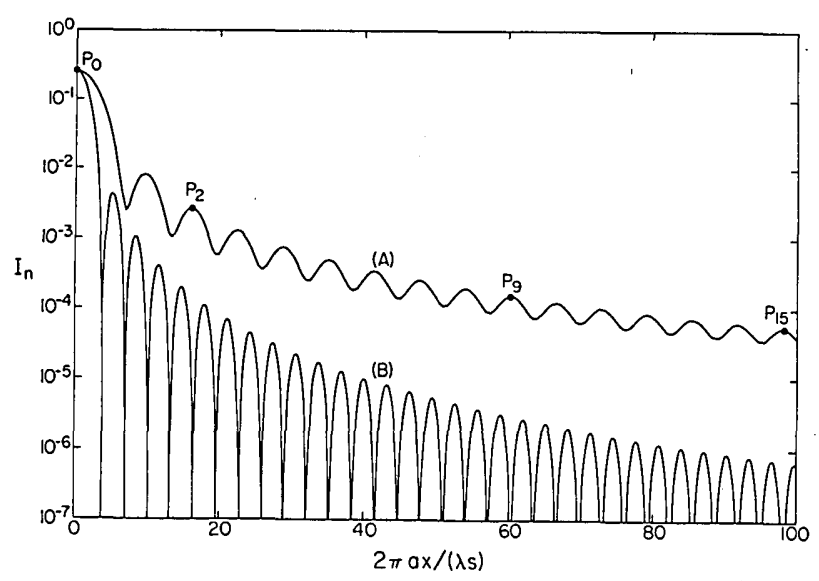

Fig. 3. Normalized transmitted irradiance $I_{n}$ is plotted logarithmically vs $\alpha x$ with $y=0$ for a semicircular aperture curve $(A)$, illuminated with a convergent wave of unit amplitude, and for a circular aperture, curve $(B)$, illuminated with a convergent wave with amplitude equal to 0.5 . 


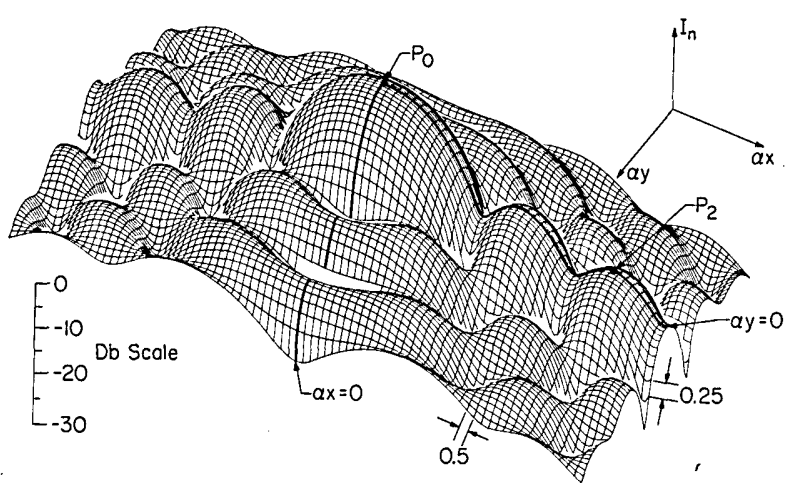

Fig. 4. Normalized transmitted irradiance $I_{n}$ is plotted logarithmically vs $\alpha x$ and $\alpha y$ with $d=0$, for a semicircular aperture illuminated with a convergent unit amplitude wave. $P_{0}$ corresponds to a normalized irradiance of 0.25 , and the range in $\alpha y$ is from -10 to +10 , and in $\alpha x$ from -20 to 20 .

Comparing $(A)$ and $(B)$ in Fig. 3, we note that the nulls are filled in when the edge is present, the intensity at large $x$ is orders of magnitude higher, and the relative maxima occur at approximately one half the frequency of that for the open aperture.

Now consider the radiation pattern for the semicircular aperture along the $y$ axis. By Eq. (17), with $x$ $=0$, we have the result

$$
\begin{aligned}
V(0, y, z=s)= & (i / \lambda s) \exp (-i k s) \\
& \times \exp \left[-i \pi y^{2} /(\lambda s)\right] \pi a^{2}\left[J_{1}(\alpha|y|) / \alpha|y|\right],
\end{aligned}
$$

which gives an intensity identical to Eq. (19) when $x$ $=0$. The significance of this is that the curve $(B)$ in Fig. 3, introduced for comparing the departure of the $x$-axis pattern caused by the edge, serves also to describe the intensity along the $y$ axis when the edge is present.

In general terms, it is clear that the intensity $V V^{*}$ computed from Eq. (16) for the offset edge has polar symmetry. This follows from noting by Eqs. (8) and (10) that $I_{1}+I_{2}$ is the Fourier transform of a realvalued function. This polar symmetry can also be shown by a direct calculation using Eq. (16).

The most interesting departure of the diffraction pattern caused by the edge occurs for $y=0$; hence we write some explicit formulas for this case. From Eqs. (17) and (18), combining the $J_{2 n+1}(\alpha y) / \alpha y$ terms, taking the limit as $y$ goes to zero, regrouping the Bessel summation, and finally using the recurrence formula AS9.1.27, we obtain the following result:

$$
\begin{aligned}
V(x, 0, z= & s)=\frac{i}{\lambda s} \exp (-i k s) \exp \left[-i \pi x^{2} /(\lambda s)\right] \pi a^{2} \\
& \times\left[\frac{J_{1}(\alpha x)}{\alpha x}+\frac{4 i}{\pi \alpha x} \sum_{m=1}^{\infty}\left(\frac{4 m^{2}}{4 m^{2}-1}\right) J_{2 m}(\alpha x)\right] .
\end{aligned}
$$

In Eq. (20), the first and second terms are even and odd-valued functions of $x$, respectively. From AS12.1.20 using AS9.1.46, we note that the second member of Eq. (20) can be expressed in terms of the first-order Struve function, i.e.,

$$
\frac{4 i}{\pi \alpha x} \sum_{m=1}^{\infty}\left[\frac{4 m^{2}}{4 m^{2}-1}\right] J_{2 m}(\alpha x)=\frac{i}{\alpha x} \mathfrak{K}_{1}(\alpha x),
$$

in which $\mathfrak{H}_{1}(\alpha x)=\mathfrak{K}_{1}(-\alpha x)$. For larger values of $|\alpha x|$, by AS12.1.31 and 9.1.3, this second member can be expressed as follows:

$$
\begin{aligned}
& \frac{4 i}{\pi \alpha x} \sum_{m=1}^{\infty}\left[\frac{4 m^{2}}{4 m^{2}-1}\right] J_{2 m}(\alpha x)=-\frac{J_{1}(\alpha x)}{\alpha x} \\
& \quad+\frac{H_{1}^{(1)}(|\alpha x|)}{\alpha x}+\frac{2 i}{\pi \alpha x}\left[1+\frac{1}{(\alpha x)^{2}}-\frac{3}{(\alpha x)^{4}}+\ldots\right]
\end{aligned}
$$

where $H_{1}{ }^{(1)}$ is the first-order Hankel function of the first kind. This expression is valid for $\pm x$ with the use of the $|\alpha x|$ argument in the Hankel function. This second member has been written explicitly, since it has a direct physical interpretation, but we should note, too, comparing Eqs. (20) and (22), the exact cancellation of the Airy disk term.

Both Eqs. (17) and (20) are well suited for numerical calculations, since algorithms for Bessel functions are readily available and since the summations can be truncated when the index exceeds the argument. However, it is of academic interest to present a solution formulated in terms of the Struve functions. Returning to Eq. (12), but setting $d=0$, we note that the limits of integration for the first integral extend from 0 to 1 while the second integral vanishes. Expanding $\sin \left[\alpha y\left(1-u^{2}\right)^{1 / 2}\right]$ in a power series and integrating term by term using AS12.1.6 lead to the following expressions for $I_{2}$ and $V$ :

$$
I_{2}=\pi a^{2} \frac{i}{\alpha x} \sum_{m=0}^{\infty} \frac{\left(-\frac{\alpha y^{2}}{4 x}\right)^{m}}{m !} \mathfrak{K}_{m+1}(\alpha x)
$$

and

$$
\begin{aligned}
V(x, y, z=s) & \dot{\cdot}=\frac{i}{\lambda s} \exp (-i k s) \exp \left[-i \pi\left(x^{2}\right.\right. \\
\left.\left.+y^{2}\right) /(\lambda s)\right] & \pi a^{2}\left\{\frac{J_{1}\left[\alpha\left(x^{2}+y^{2}\right)^{1 / 2}\right]}{\alpha\left(x^{2}+y^{2}\right)^{1 / 2}}\right. \\
& \left.+\frac{i}{\alpha x} \sum_{m=0}^{\infty} \frac{\left(-\frac{\alpha y^{2}}{4 x}\right)^{m}}{m !} \mathfrak{H}_{m+1}(\alpha x)\right\} .
\end{aligned}
$$

Except for special cases, this expression is not particularly convenient for numerical calculations.

Three-dimensional computer plots are shown in

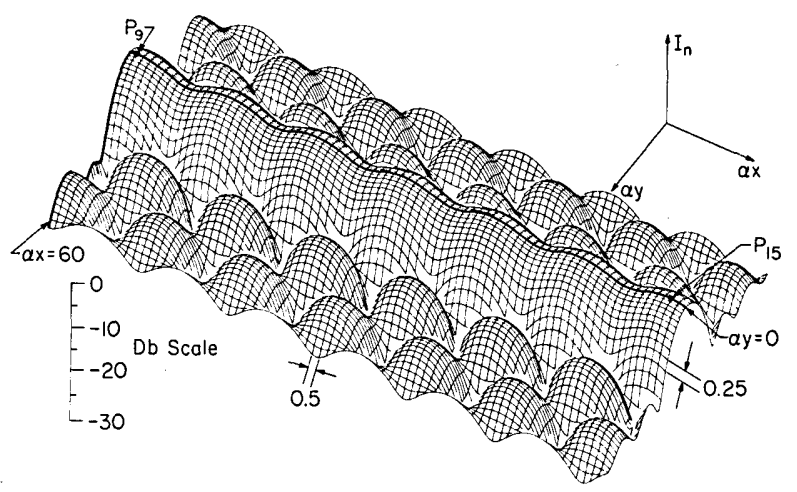

Fig. 5. Normalized transmitted irradiance $I_{n}$ plotted logarithmically vs $\alpha x$ and $\alpha y$ with $d=0$, for a semicircular aperture illuminated with a convergent unit amplitude wave. The interval along the $\alpha y$ axis is from $-10 \leq \alpha y \leq 10$, and $\alpha x$ spans the relative maxima from $P_{9}$ through $P_{15}$; the point $P_{9}$ corresponds to a normalized irradiance of $1.4 \times 10^{-4}$. 


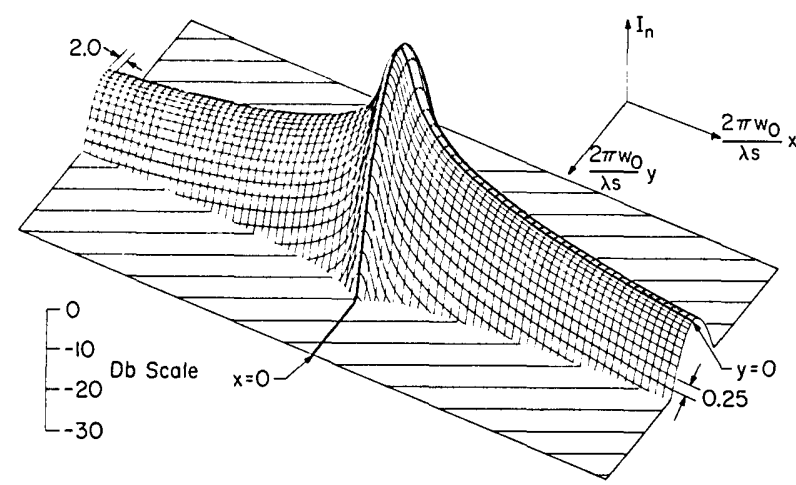

Fig. 6. Normalized transmitted irradiance $I_{n}$ is plotted logarithmically vs $2 \pi w_{0} y /(\lambda s)$ and $2 \pi w_{0} x /(\lambda s)$ with $d=0$, for an infinite edge illuminated with a convergent Gaussian wave. The maximum of $I_{n}$ corresponds to a normalized irradiance of 1.0 while the horizontally ruled plane corresponds to an irradiance level of $10^{-5}$. The interval along the normalized $x$ axis is from $-80 \leq 2 \pi w_{0} x / s \leq$ 80 and along $y$ from $-10 \leq 2 \pi w_{0} y / \lambda s \leq 10$.

Figs. 4 and 5 for a semicircular aperture illuminated by a converging wave of uniform amplitude. The center point of the radiation pattern is labeled $P_{0}$ and shown both in Figs. 3 and 4 . Likewise, to provide orientation, the second relative maximum along $x$ is labeled $P_{2}$. The polar symmetry of the intensity pattern is evident, and this is seen, too, in Fig. 1(b). A logarithmic intensity scale is used, as labeled, with $P_{0}$ corresponding to a normalized absolute intensity of 0.25 . Contour intervals in $\alpha x$ of 0.5 and in $\alpha y$ of 0.25 are used. Thus, in Fig. 4, the entire plot encompasses the region $-20<\alpha x<20$ and $-10<\alpha y<10$.

Now the details of the radiation spike at larger values of $\alpha x$ are shown in Fig. 5. The span in $\alpha x$ encompasses the relative maxima from $P_{9}$ through $P_{15}$; and the contour intervals and logarithmic intensity scale are as described for Fig. 4. The interval along the $y$ axis is from $-10<\alpha y<10$.

\section{Edge Diffraction of a Convergent Gaussian Wave}

\section{Theory}

Now, consider a converging Gaussian wave incident on an infinite edge offset by the distance $d$ along the $\xi$ axis. Equation (4) is rewritten for this incident wave as follows:

$$
U_{g}(\xi, \eta)=\exp \left[i \pi\left(\xi^{2}+\eta^{2}\right) /(\lambda s)-\left(\xi^{2}+\eta^{2}\right) / w_{0}^{2}\right],
$$

where $w_{0}$ is the radius at which the incident intensity has dropped to its $1 / e^{2}$ value. For a transmission function that is simply an edge, we write

$$
T(\xi, \eta)=\frac{1}{2}[1+\operatorname{sgn}(\xi-d)] .
$$

Equations (25) and (26) are multiplied, giving the assumed field distribution $U^{\prime}=T U_{g}$, which is substituted in Eq. (5) and integrated using AS7.4.32 and 7.1.16. The result is given by

$$
\begin{aligned}
V_{g}(x, y, z= & s)=[i /(2 \lambda s)] \exp (-i k s) \exp \left[-i \pi\left(x^{2}\right.\right. \\
+ & \left.\left.y^{2}\right) /(\lambda s)\right] \cdot \pi w_{0}^{2} \exp \left[-\left(\pi w_{0} / \lambda s\right)^{2}\left(x^{2}\right.\right. \\
& \left.\left.+y^{2}\right)\right]\left\{1-\operatorname{erf}\left[\left(d / w_{0}\right)-\left(i \pi x w_{0} / \lambda s\right)\right]\right\},
\end{aligned}
$$

where erf $z$ is given by AS7.1.1. This expression is applicable for positive and negative values of $d$. As a consistency test of Eq. (27), letting $d \rightarrow-\infty$, i.e., no edge, we note that the erf $(-\infty)=-1$, and Eq. (27) reduces to a well-known result. Also, this expression is functionally similar to the result of Pearson et al., who studied the diffraction of Gaussian beams by a semiinfinite plane. ${ }^{5}$ Specifically, Eq. (8) in Ref. 5 can be considered in the limit at $z \rightarrow \infty$, i.e., the transform or back focal plane distribution is a scaled far-zone radiation pattern.

By AS7.1.3, Eq. (27) is rewritten in terms of the $w$ function as follows:

$$
\begin{aligned}
V_{g}(x, y, z=s)=[i /(2 \lambda s)] \exp (-i k s) \exp \left[-i \pi\left(x^{2}\right.\right. \\
\left.\left.+y^{2}\right) /(\lambda s)\right] \exp [i 2 \pi x d /(\lambda s)] \cdot \pi w_{0}^{2} \exp \left\{-\left[\pi w_{0} y /(\lambda s)\right]^{2}\right. \\
\left.-\left(d / w_{0}\right)^{2}\right\} w\left[\pi w_{0} x /(\lambda s)+i d / w_{0}\right] .
\end{aligned}
$$

For small $d / \mathrm{w}_{0}$ and large values of $\pi w_{0} x /(\lambda s)$ by Ref. 11, p. 328, we note that Eq. (28) can be expressed as follows:

$$
\begin{aligned}
& V_{g}(x, y, z=s)=[1 /(\lambda s)] \exp (-i k s) \exp \left[-i \pi\left(x^{2}\right.\right. \\
& \left.\left.+y^{2}\right) /(\lambda s)\right] \exp [i 2 \pi x d /(\lambda s)] \cdot \pi w_{0}{ }^{2} \exp \left\{-\left[\pi w_{0} y /(\lambda s)\right]^{2}\right\}\left(d / w_{0}\right. \\
& -1)\left\{0.256 /\left[\pi w_{0} x /(\lambda s)\right]\right\} . \quad(29)
\end{aligned}
$$

For an aperture extending from $\xi=d_{1}$ to $\xi=d_{2}$ with $d_{1}<d_{2}$, i.e., an infinite slit, by Eq. (27), it is readily shown that the electric field $V_{s g}$ is given by

$$
\begin{aligned}
& V_{s g}(x, y, z=s)=[i /(2 \lambda s)] \exp (-i k s) \exp \left[-i \pi\left(x^{2}\right.\right. \\
& \left.\left.+y^{2}\right) /(\lambda s)\right] \cdot \pi w_{0}^{2} \exp \left[-\left(\pi w_{0} / \lambda s\right)^{2}\left(x^{2}+y^{2}\right)\right]\left\{\operatorname { e r f } \left[\left(d_{2} / w_{0}\right)\right.\right. \\
& \left.\left.-\left(i \pi x w_{0} / \lambda s\right)\right]-\operatorname{erf}\left[\left(d_{1} / w_{0}\right)-\left(i \pi x w_{0} / \lambda s\right)\right]\right\} .
\end{aligned}
$$

\section{Computations and Discussion}

A three-dimensional plot for the diffraction of a convergent Gaussian wave by an edge is shown in Fig. 6. $V_{g} V_{g} *$ is calculated from Eq. (28) and plotted logarithmically vs $2 \pi w_{0} x /(\lambda s)$ and $2 \pi w_{0} y /(\lambda s)$ with $d$

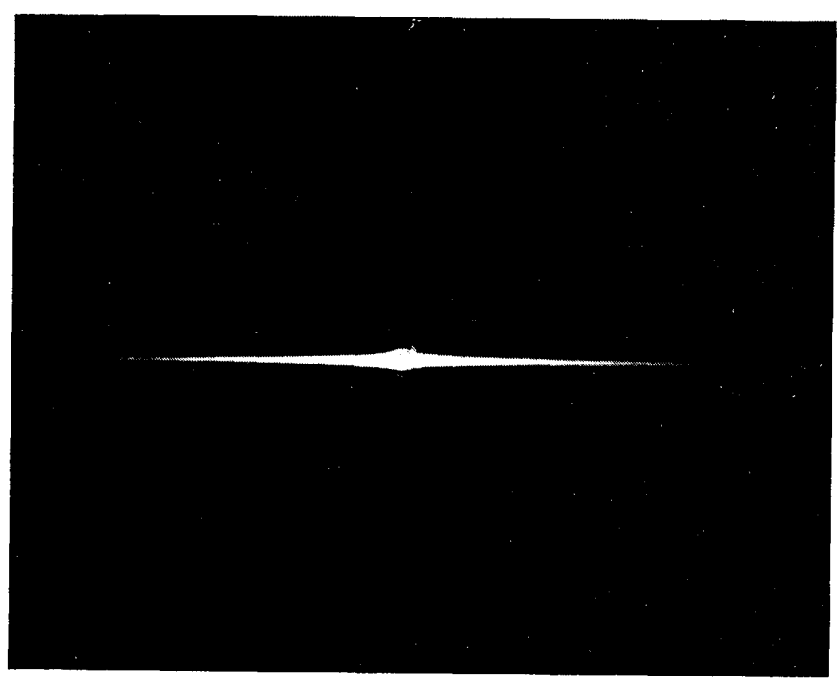

Fig. 7. Recorded intensity for a convergent wave of Gaussian amplitude truncated by an infinite edge. 


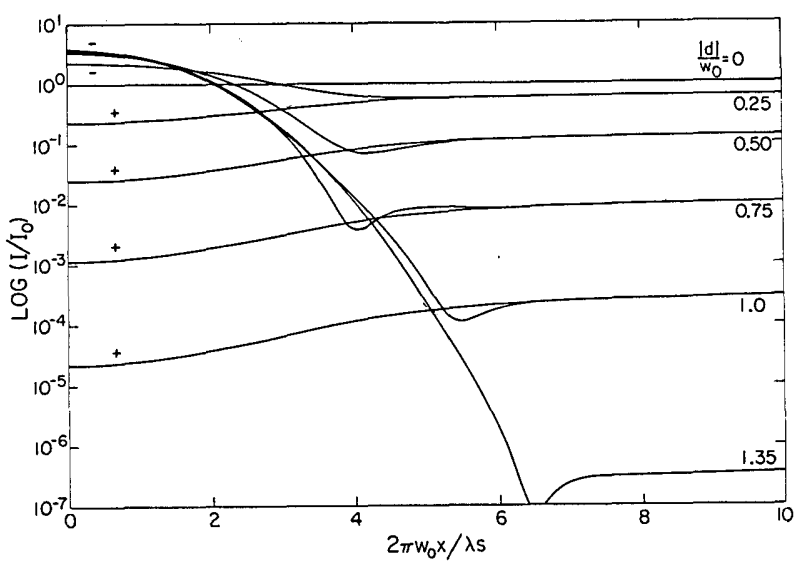

Fig. 8. Transmitted irradiance $I$ normalized to its $d / w_{0}=0$ value is plotted logarithmically vs $2 \pi \omega_{0} x /(\lambda s)$ for $y=0$. The curves whose normalized intensity at $2 \pi w_{0} x /(\lambda s)=0$ is greater than one correspond to negative values of $d / w_{0}$.

Table I. Nulls of Radiation Pattern for a Convergent Gaussian Wave Diffracted by an Offset Edge, Eq. (28)

\begin{tabular}{cc}
\hline$d / w_{0}$ & $\pi w_{0} x /(\lambda s)$ \\
\hline-1.355 & 1.991 \\
-2.177 & 2.691 \\
-2.784 & 3.235 \\
-3.287 & 3.697 \\
-3.726 & 4.106 \\
\hline
\end{tabular}

$=0$. Comparing Figs. 4, 5, and 6, we note that the spike in the diffraction pattern for the Gaussian drops off smoothly without the ringing observed in the former case. An edge transform pattern is shown in Fig. 7 for Gaussian illumination with $2 \pi w_{0} /(\lambda s)=$ 35.1 for comparison to that for the uniformly illuminated semicircular aperture with $2 \pi a /(\lambda s)=58.5$ [see Fig. 1(b)].

A graphic understanding of the variation in the phase and amplitude of the Gaussian wave diffracted by an edge can be obtained from the contour line diagram for the $w$ function, Ref. 11, Fig. 7.3. For example, a fixed value of $d / w_{0}$ sets the imaginary part of the $w$ function. The variation in $|w|$ is easily read as a function of the real part that corresponds to displacement in the transform plane in units of $\pi w_{0} x /(\lambda s)$. As $d$ in Eq. (28) goes negative, the edge is exposing more of the Gaussian wavefront and interesting nulls are to be expected at zeros of the $w$ function (see Table I). The first null is seen also in the curve in Fig. 8 for the case $d / \mathrm{w}_{0}=-1.35$.

The variation in intensity vs normalized displacement along $x$, with $y=0$ and for the parameter $d /$ $w_{0}$, is shown in Fig. 8. Plotted logarithmically is the intensity $V_{g} V_{g} *\left[2 \pi w_{0} x /(\lambda s), y=0, z=s ; d / w_{0}\right]$ normalized to its $d / w_{0}=0$ value as given in Eq. (28). Values for negative $d / w_{0}$, exposing more aperture, are seen to have larger central intensity, peaking in the limit to a normalized value of $V_{g} V_{g}$ * equal to 4 . Asymptotically, the curves for $\pm d / w_{0}$ merge as $2 \pi w_{0} x /(\lambda s)$ exceeds 6 .

\section{Summary and Conclusions}

Several cases of edge diffraction of convergent spherical waves have been analyzed. The resulting electric field for the case of an infinite edge, positioned off-center in a circular aperture, is given in Eq. (16). The solution is particularized to the focal plane of the convergent wave. The corresponding result for a $\pm \pi$-radian phase plate can be written directly from Eqs. (7) and (13).

The dominant feature of the focal plane pattern due to the edge is the large spike of energy diffracted at right angles to the edge. Qualitatively, this effect is well known; in frequency plane analysis, high frequency content is often used as an indicator of edge sharpness, hence image quality, in photogrammetry. Having precise formulas that are appropriate for numerical calculations, e.g., Eq. (17), should be useful in such applications. To illustrate these calculations, we present $3-D$ plots of the intensity for a region near the origin and out along the spike. Graphs are shown in Fig. 3 for the intensity profile along the $x$ and $y$ axes.

The optical transform pattern for a convergent Gaussian wave diffracted by an edge is also analyzed. The singularities that customarily occur in diffraction pattern problems without an aperture are absent in this case, as is to be expected with Gaussian beams. The result for an offset edge is given in Eq. (27), while that for an asymmetrical slit is presented in Eq. (30).

Characteristically, it has been found that the intensity in the spike due to the edge falls off asymptotically as $1 / x^{2}$, where $x$ is the distance from the focal point. For the circular aperture, there is a fractional ripple even at large values of $x$, but without sharp nulls. Quantitatively, the fractional ripple is seen by Eq. (22) to be on the order of $\left|H_{1}^{(1)}(|\alpha x|)\right|^{2}$. However, with Gaussian beam illumination, the ripple vanishes, as is seen in Fig. 8, although interesting nulls are predicted at large offsets of the edge as given in Table I.

This research was supported by the Air Force Office of Scientific Research.

\section{References}

1. A. Sommerfeld, Optics (Academic Press, New York, 1967).

2. J. B. Keller, J. Opt. Soc. Am. 52, 116 (1962).

3. W. Braunbek and G. Laukien, Optik 9, 174 (1952).

4. F. E. Borgnis and C. H. Papas, Randwertprobleme Der Mikrowellenphysik (Springer-Verlag, Berlin, Göttingen, 1955), pp. 101-157.

5. J. E. Pearson, T. C. McGill, S. Kurtin, and A. Yariv, J. Opt. Soc. Am. 59, 1440 (1969).

6. H. Lipson, Optical Transforms (Academic Press, London, 1972).

7. E. Lommel, Abh. Bayer Akad. 15, Abth, 2, 233, (1885).

8. H. Struve, Mem. Acad. St. Petersbourgh 34, 1 (1886).

9. H. Linfoot and E. Wolf, Proc. Phys. Soc. B 69, 823 (1956).

10. J. W. Goodman, Introduction to Fourier Optics (McGrawHill, New York, 1968).

11. M. Abramowitz and I. A. Stegun, Eds. Handbook of Mathematical Functions (Natl. Bureau of Standards, Washington, D.C., 1970). 